\title{
Modified SIR Compartmental Epidemic Model with Social Distancing and Hospital Saturation Applied to the COVID-19 Pandemic
}

\author{
V.R. da Silva, O. H. Menin
}

The rapid spread of SARS-CoV-2/COVID-19 in the first months of 2020 overburdened health systems worldwide. The absence of vaccines led public authorities to respond to the pandemic by adopting nonpharmaceutical interventions, mainly social distancing policies. Yet concerns have been raised on the economic impact of such measures. Considering the impracticability of conducting controlled experiments to assess the effectiveness of such interventions, mathematical models have played an essential role in helping decision makers. Here we present a simple modified SIR (susceptible-infectious-recovered) model that includes social distancing and two extra compartments (hospitalized and dead due to the disease). Our model also incorporates the potential increase in the mortality rate due to the health system saturation. Results from numerical experiments corroborate the striking role of social distancing policies in lowering and delaying the epidemic peak, thus reducing the demand for intensive health care and the overall mortality. We also probed into optimal social distancing policies that avoid the health system saturation and minimize the economic downturn.

Keywords: epidemiology, infectious diseases, SARS-CoV-2, mathematical modeling, computational simulation, differential equations

\section{Introduction}

By the end of 2020, the severe acute respiratory syndrome-coronavirus 2 (SARS-Cov-2/ COVID-19) pandemic had affected nearly 80 million people worldwide and claimed over 1.7 mil-

Received April 7, 2021

Accepted July 7, 2021

Programa Institucional de Bolsas de Iniciação Cinentífica do IFSP (PIBIFSP) (Edital SRT.18/2019)

Vinicius R. da Silva

viniciysrod@gmail.com

Olavo H. Menin

olavohmenin@ifsp.edu.br

Instituto Federal de Educação, Ciência e Tecnologia de São Paulo

R. Américo Ambrósio, 269 - Jardim Canaa, Sertãozinho - SP, 14169-26, Brazil

RUSSIAN JOURNAL OF NONLINEAR DYNAMICS, 2021, 17(3), 275-287 
lions lives [3]. Its index case was reported late December 2019 in Wuhan, Southeast China. Despite the first containment measures taken by the Chinese government to halt the spread [4], by the end of January 2020 the virus had already reached 7818 cases in 19 countries forcing the World Health Organization (WHO) to classify the outbreak as a public emergency of international concern. At that moment, Italy and Spain, countries that would head the list of COVID-19 death toll had no cases reported yet and the United States, the country currently most burdened by the pandemic, had only 5 confirmed cases [5]. On March 11, with cases and deaths soaring on virtually all continents, the "two-month outbreak" was characterized as a pandemic by WHO [6].

SARS-CoV-2 is an RNA virus that belongs to the same genus of SARS-CoV-1 and MERSCoV coronaviruses, respectively responsible for the 2002-2003 Severe Acute Respiratory Syndrome outbreak in Asia and the Middle East Respiratory Syndrome reported in Arabian Peninsula in 2012 [7, 8]. While most individuals infected with SARS-CoV-2 are asymptomatic, a significant fraction develop severe respiratory problems and need to be hospitalized in Intensive Care Unit (ICU) to receive mechanical ventilation support. The lack of knowledge about the disease, combined with its fast spread, have led to the collapse of the health systems in several countries, bringing up issues such as mechanical ventilators shortage and medical professionals burnout $[9,10]$.

Within this scenario and without vaccines against the virus, the early responses taken by most public authorities were based on nonpharmaceutical interventions (NPIs), predominantly travel restriction, closure of schools and universities, social distancing and even national lockdowns. The main idea was to give health systems extra time to get prepared once low mobility means less daily contacts, which can reduce the transmission and delay the epidemic peak, i. e., "flatten the curve" $[11,12]$. In short, the goal was to reduce the effective reproduction number, $R_{t}$, which represents the average number of infections produced by a single infected individual in a partially immune population $[13,14]$. However, governments have also raised concerns about the benefit-cost of these measures due to their social and economic impacts $[15,16]$ and some have even denied the severity of the disease [17]. Decision makers must then be provided optimal policies options that minimize both the burden of the disease and the economic impact caused by NPIs [11].

Taking into account the impossibility of performing controlled experiments to assess the effectiveness of a given intervention, mathematical models have played an essential role in the war against the pandemic [18]. Nakamura et al. [19] proposed a simple discrete SIR model and assessed how different confinement strategies affect an eventual epidemic second wave. Pedro et al. [20] also investigated the emergence of the second wave but proposing a compartmental model coupled with imitation dynamics. Block et al. [21] analyzed strategies based on social networks to assess how clusterization affects the effectiveness of social isolation. A Bayesian approach was applied by Flaxman et al. [22] to evaluate how different NPIs strategies affect the effective reproduction number, $R_{t}$, in European countries. A stochastic compartmental model, on the other hand, was used by Kucharski et al. [23] to capture the impact of travel restriction on the virus spread during the first month of the epidemic in China.

Here we present a simple modified SIR (susceptible-infectious-recovered) model that considers social distancing and two extra compartments, one encompassing hospitalized individuals in ICU who need mechanical ventilation support, and other counting those who die due to the disease. Our model also takes into account the eventual increase in the death rate due to ICU shortage. The main goal of our study is to investigate the role of social isolation policies on both the need of ICU and the mortality due to the disease. Numerical experiments considering different scenarios were carried out and the results highlight the central importance of suitable 
social isolation policies in both lowering and delaying the epidemic peak, thus reducing the need for ICU and providing governments more time to prepare the health systems. More specifically we sought for scenarios that present optimal social distancing policies that minimize both deaths due to ICU shortage and impact on the economy. Although the model could have incorporated the disease latent period and vital dynamics, for simplicity we chose not to do so once initial assessment showed negligible impact of these elements on the main results.

\section{SIRHD model}

The model we present here considers no vital dynamics and is based on the classical SIR model originally proposed by W. Kermack and A. McKendrick [24], which classifies the host population in three compartments, susceptible (S), infectious (I) and recovered (R). On the other hand, since our model is meant to bring to light some features of the SARS-Cov-2/COVID-19 pandemic, we consider social distancing ("social isolation") and two extra compartments, namely, the hospitalized individuals in ICU requiring mechanical ventilation support $(\mathrm{H})$ and those who die due to the disease (D). We hereafter refer to our model as SIRHD and it is represented in Fig. 1.

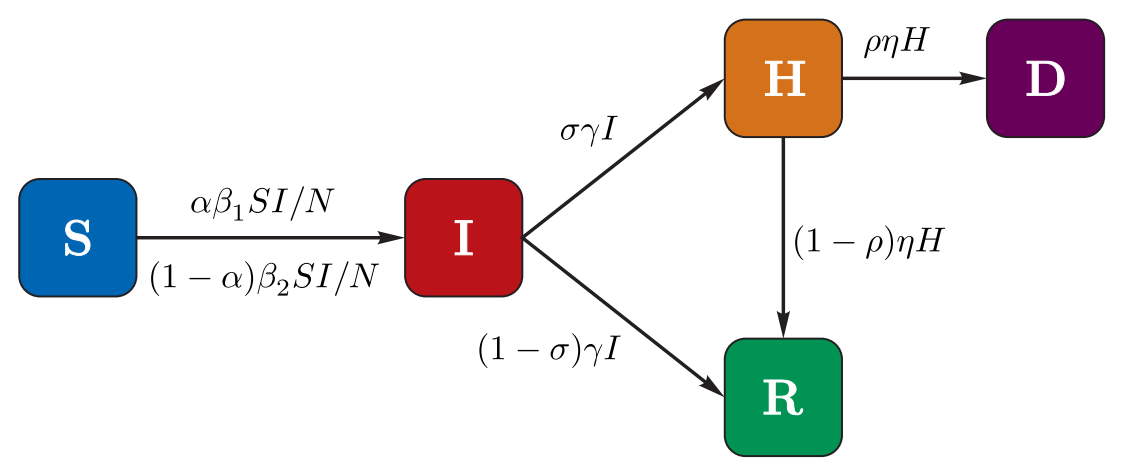

Fig. 1. Compartments of the SIRHD model: S (susceptible), I (infectious), R (recovered), H (hospitalized) and $\mathrm{D}$ (dead). The role of the parameters $\beta_{1}, \beta_{2}, \gamma, \sigma$ and $\eta$ and functions $\alpha(t)$ and $\rho(H)$ are described in the text and summarized in Table 1.

Let $S(t), I(t), R(t), H(t)$ and $D(t)$ be the absolute number of individuals in the compartments $\mathrm{S}, \mathrm{I}, \mathrm{R}, \mathrm{H}$ and $\mathrm{D}$ at a given time $t$, respectively, and $N=S(t)+I(t)+R(t)+H(t)+D(t)$ the size of the population. At the beginning of the epidemic $(t=0)$, all individuals excepting the index case (the first infectious individual) start as susceptible. As time goes by, susceptible individuals can be infected when they contact infectious ones. Commonly the rate at which it occurs is $\beta S(t) I(t) / N$, with the transmission coefficient $\beta$ depending on both the average number of contacts an individual has and the probability of transmission given contact [25]. In our model, we define two transmission coefficients, $\beta_{1}$, for individuals under social isolation, and $\beta_{2}$, otherwise, with $\beta_{1}<\beta_{2}$. If we let $\alpha(t) \in[0,1]$ be the social isolation level, namely, the fraction of individuals practicing social distancing, the $\mathrm{S} \rightarrow \mathrm{I}$ transition rate is $\alpha(t) \beta_{1} S(t) I(t) / N+(1-$ $-\alpha(t)) \beta_{2} S(t) I(t) / N$. Once infected, a fraction $\sigma \in[0,1]$ of the individuals need to be hospitalized in ICU (requiring mechanical ventilation support), the rest getting recovered. Hence, $\gamma$ being the inverse of the infectious period, the $\mathrm{I} \rightarrow \mathrm{H}$ and $\mathrm{I} \rightarrow \mathrm{R}$ transition rates are given by $\sigma \gamma I(t)$ and $(1-\sigma) \gamma I(t)$, respectively. Lastly, considering that a fraction $\rho(H) \in[0,1]$ of the hospitalized individuals die and the remaining recover, the transitions $\mathrm{H} \rightarrow \mathrm{D}$ and $\mathrm{H} \rightarrow \mathrm{R}$ 
occur at rates $\rho(H) \eta H$ and $(1-\rho(H)) \eta H$, respectively, where $\eta$ is the inverse of the average hospitalization period. The SIRHD model is then governed by the system of ordinary differential equations

$$
\begin{aligned}
& \frac{d S(t)}{d t}=-\alpha(t) \beta_{1} S(t) \frac{I(t)}{N}-(1-\alpha(t)) \beta_{2} S(t) \frac{I(t)}{N}, \\
& \frac{d I(t)}{d t}=\alpha(t) \beta_{1} S(t) \frac{I(t)}{N}+(1-\alpha(t)) \beta_{2} S(t) \frac{I(t)}{N}-\gamma I(t), \\
& \frac{d H(t)}{d t}=\sigma \gamma I(t)-\eta H(t), \\
& \frac{d D(t)}{d t}=\rho(H) \eta H(t), \\
& \frac{d R(t)}{d t}=(1-\sigma) \gamma I(t)+(1-\rho(H)) \eta H(t) .
\end{aligned}
$$

Analogous to the effective reproduction number, $R_{t}$, the basic reproductive number, $R_{0}$, is defined as the average number of secondary cases arising from a primary case in an entirely susceptible population. Commonly used to gauge the beginning of an outbreak, its value depends on both the disease itself and the host population characteristics and it is related to the threshold phenomenon: If $R_{0}>1$, the pathogen invades the population and thus the epidemic occurs, otherwise the epidemic dies out [25]. Moreover, it is associated with the concept of "herd immunity", namely, when a population is provided with protection against an infectious disease due to an immune fraction of its individuals. For a well-mixed population, for instance, such a fraction is $1-1 / R_{0}$ [26]. Specifically in our SIRHD model, and considering $\alpha(t)=\alpha$ constant in the first stages of the outbreak, the basic reproductive number is

$$
R_{0}=\frac{\alpha \beta_{1}+(1-\alpha) \beta_{2}}{\gamma}
$$

The most dramatic scenes witnessed in the first months of the COVID-19 pandemic were patients dying breathless due to the lack of mechanical ventilators. This overburden of the health system has had a high impact on the mortality rate and has been the major reason for the attempts at flattening the pandemic curve. In our model, we seek to incorporate the increase of mortality due to the shortage of ICU (with mechanical ventilator) considering $\rho(H)$ (fraction of the hospitalized individuals that die) as given by

$$
\rho(H)= \begin{cases}\rho_{0}, & H \leqslant \Omega, \\ \frac{\Omega \rho_{0}+(H-\Omega)}{H}, & H>\Omega,\end{cases}
$$

where $\rho_{0}$ is the baseline (when there is no shortage of ICU) and $\Omega$ is the available number of ICU. According to this assumption, all patients that need, but do not receive mechanical ventilation support die.

Finally, we assume for simplicity a unique period of social isolation with duration $\tau$ and level $\alpha>0$ (constant) that starts after a delay $\delta$ from the beginning of the epidemic. Hence, we define the social distancing schedule as

$$
\text { social distancing schedule } \begin{cases}\alpha(t)=0 & \text { for } 0<t<\delta, \\ \alpha(t)=\alpha>0 & \text { for } \delta<t<\delta+\tau, \\ \alpha(t)=0 & \text { for } t>\delta+\tau .\end{cases}
$$




\section{Numerical experiments and results}

The model was computationally implemented in MATLAB ${ }^{\circledR}$ pseudo-code and numerically solved by using the function ode45. The numerical experiments were designed aiming to explore how different social distancing policies affect the COVID-19 mortality. The parametrization was carried out based on the literature and on actual COIVD-19 data, mainly from Johns Hopkins University, Center for Disease Control and Prevention (CDC) and European Centre for Disease Prevention and Control (ECDC). The baseline parameters and variables adopted in the numerical experiments are depicted in Table 1.

Table 1. Baseline parameters and variables adopted in the numerical experiments.

\begin{tabular}{clc}
\hline Symbol Description & Values \\
\hline$N$ & Population size & 100000 \\
$\beta_{1}$ & Transmission coefficient for individual under social isolation & $0.1\left[\mathrm{day}^{-1}\right]$ \\
$\beta_{2}$ & Transmission coefficient for individual under no social isolation & $0.5\left[\mathrm{day}^{-1}\right]$ \\
$\gamma$ & Inverse of the infectious period & $0.2\left[\mathrm{day}^{-1}\right]$ \\
$\eta$ & Inverse of the hospitalization period & $0.2\left[\mathrm{day}^{-1}\right]$ \\
$\Omega$ & Number of available mechanical ventilators & 20 \\
$\sigma$ & Fraction of infected individuals who need ICU (requiring ventilation support) & 0.005 \\
$\rho_{0}$ & Fraction of hospitalized individuals who die & 0.1 \\
$\alpha$ & Fraction of individuals practicing social distance (social isolation level) & variable \\
\hline
\end{tabular}

The population size was chosen arbitrarily as being $10^{5}$ due to statistics about the number of cases, deaths and ICU in the literature being commonly presented as "per 100000 individuals". To set the transmission coefficient for individuals under no social isolation, $\beta_{2}$, we considered $\alpha=0$ in the early stages of the outbreak and applied Eq. (2.6) with $\gamma=0.2$ day $^{-1}$ and $R_{0}=$ $=2.5[8]$. For individuals under social isolation, on the other hand, we considered that the average number of effective daily contacts drops five times compared to those not under social isolation (from about 13.4 [27] to approximately 2.7), and therefore, set $\beta_{1}=\beta_{2} / 5$. Finally, the per capita number of ICU varies substantially around the world, ranging from 1 to 30 per 100000 inhabitants $[28,29]$ and thus we arbitrarily set $\Omega=20$.

For clarity, the numerical experiments are presented in two parts. First, we simulated scenarios in which the social isolation level, $\alpha$, is set constant during the entire epidemic. We also ascertained the influence of the quality of such an isolation considering different values of $\beta_{1}$. Second, we investigated more realistic situations considering limited periods of social isolation, i. e., adopting both a delay to its beginning and a specific time to its end. Lastly, we sought out for scenarios that provide good compromise between the potential economic impact of the social isolation and the mortality caused by ICU shortage.

\section{Numerical experiments: First part}

As a baseline scenario, we initially assumed no social distancing, i. e., we set $\alpha=0$ during the entire simulation (from $t=0$ to $t=100$ days). All other parameters followed Table 1 and the results are shown in Fig. 2, which depicts the temporal evolution of the number of individuals in each compartment. One can clearly see the familiar behavior of the susceptible (S), infectious (I) and recovered $(\mathrm{R})$ compartments as in the classical SIR model, whereas the inset shows the 
two extra compartments, hospitalized (H) and dead (D). One must note the abrupt change in the slope of the compartment D graph when the number of hospitalized reaches the ICU saturation $(\Omega=20)$. As modeled by Equation (2.7), from this point on, all individuals who need ICU, but are not provided with it, die. At the end of the epidemic, this scenario tallied over 80 thousand infected with 295 death, 278 of which were caused by lack of ICU.

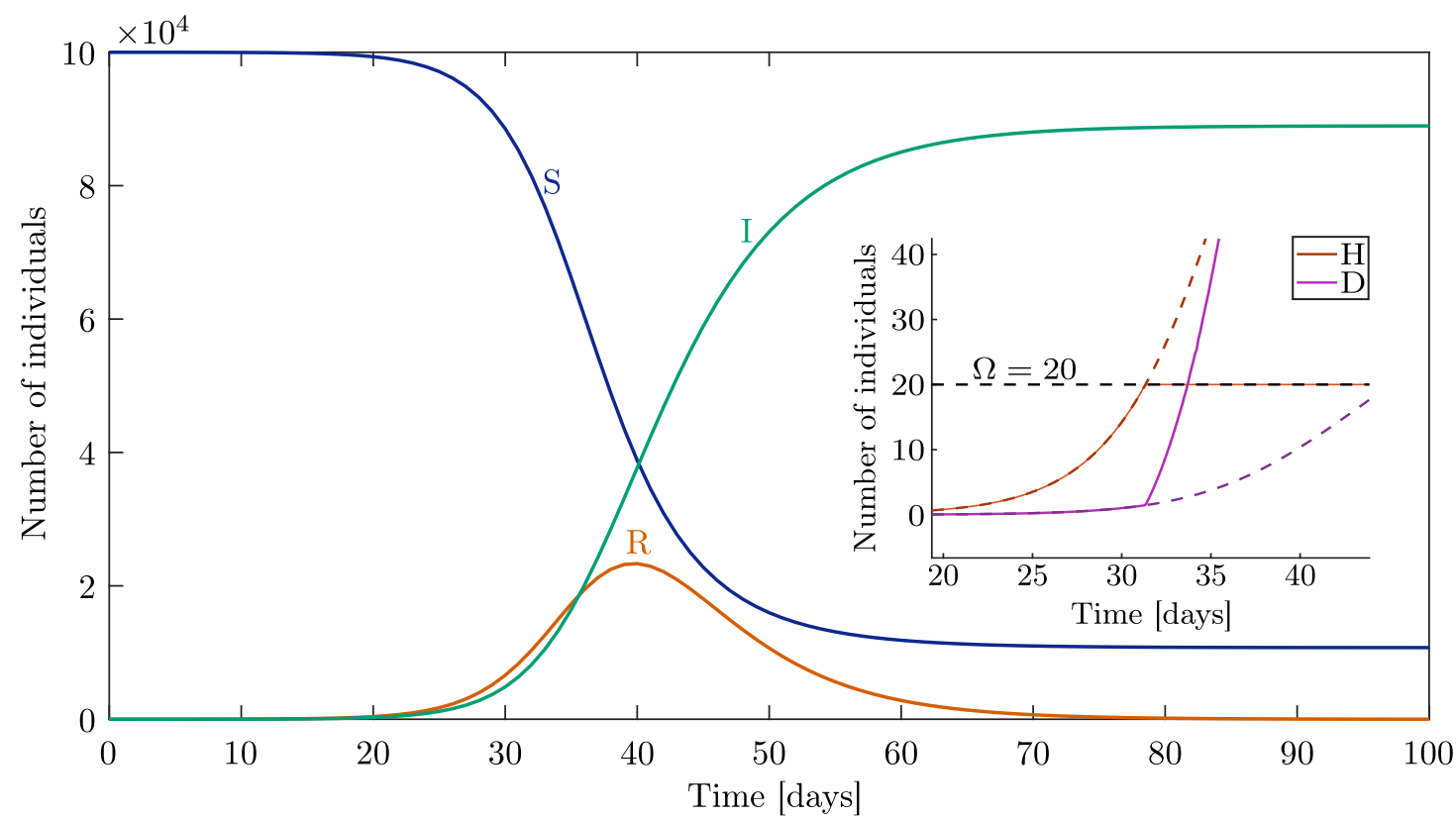

Fig. 2. Baseline scenario with no social isolation $(\alpha=0)$ : Temporal evolution of the number of individuals in the compartments $\mathrm{S}$ (susceptible), I (infectious), R (recovered), $\mathrm{H}$ (hospitalized) and D (dead). The horizontal dashed line represents the number of ICU available $(\Omega=20)$. The simulations were run following the parameters in Table 1.

In the following, we sought to capture the overall effect of the social isolation on both the ICU demand and the death toll. For this we simulated scenarios with different social isolation levels, $\alpha=0.15, \alpha=0.30, \alpha=0.45$ and $\alpha=0.60$, all kept constant during the entire epidemic. The other parameters followed Table 1 . Undoubtedly these scenarios are unfeasible since they result in high economic impact. Despite this, the results show a remarkable effect of the social distancing policies in lowering and delaying the ICU demand and, hence, saving lives, as depicted in Fig. 3 and Table 2. Specifically, we found that for $\alpha \approx 0.57$ there will not be ICU shortage (once the isolation is kept until the end of the epidemic).

Table 2. Maximum ICU demand, total deaths and death due to ICU shortage considering different social isolation levels, $\alpha$, all kept constant during the entire epidemic.

\begin{tabular}{cccc}
\hline$\alpha$ & Maximum ICU demand Total deaths Deaths due to ICU shortage \\
\hline 0 & 98 & 295 & 278 \\
0.15 & 81 & 256 & 238 \\
0.30 & 62 & 198 & 178 \\
0.45 & 38 & 108 & 84 \\
0.60 & 14 & 21 & 0 \\
\hline
\end{tabular}




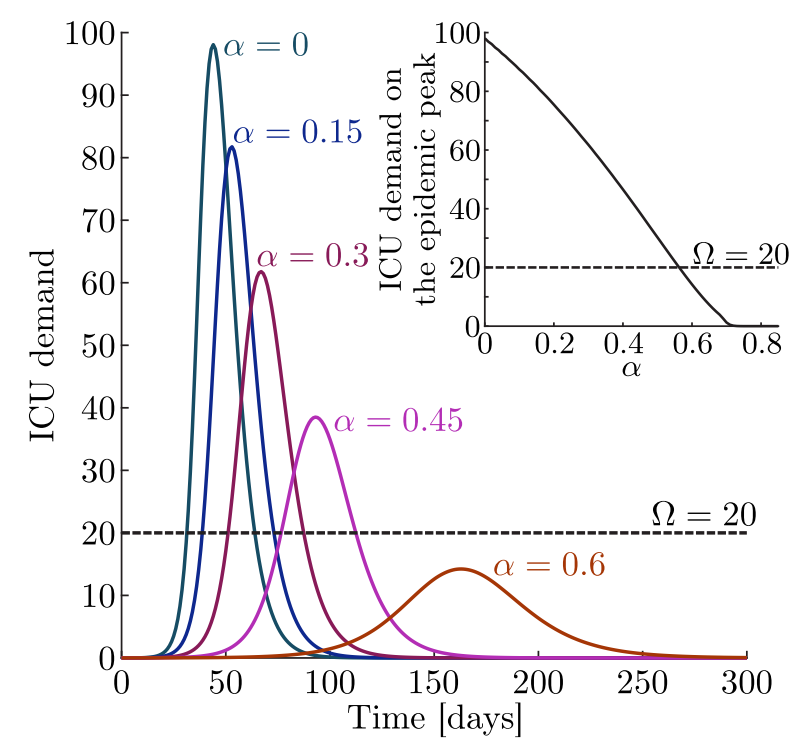

(a)

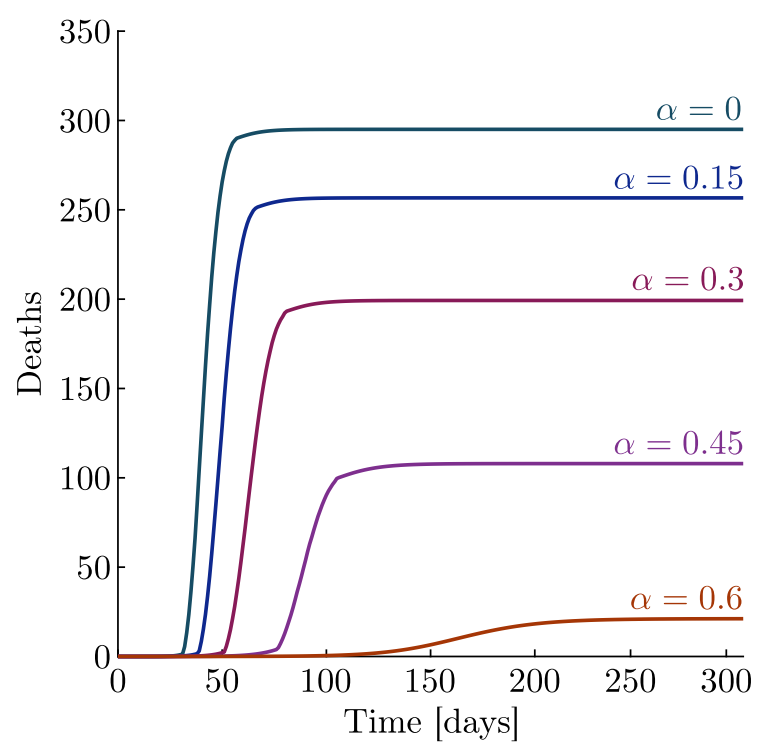

(b)

Fig. 3. Temporal evolution of the (a) ICU demand and (b) number of deaths for different values of social isolation level $\alpha$, maintained constant during the entire epidemic. The simulations were run following the parameters in Table 1.

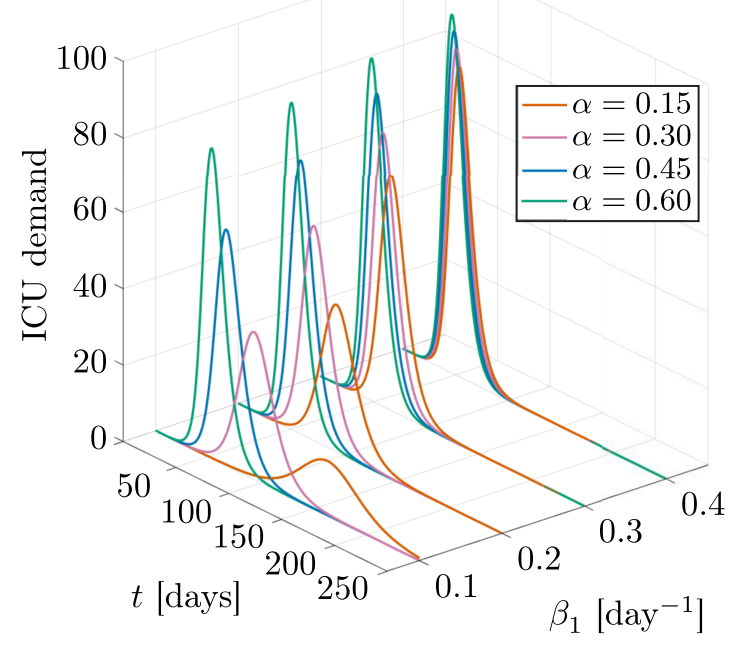

(a)

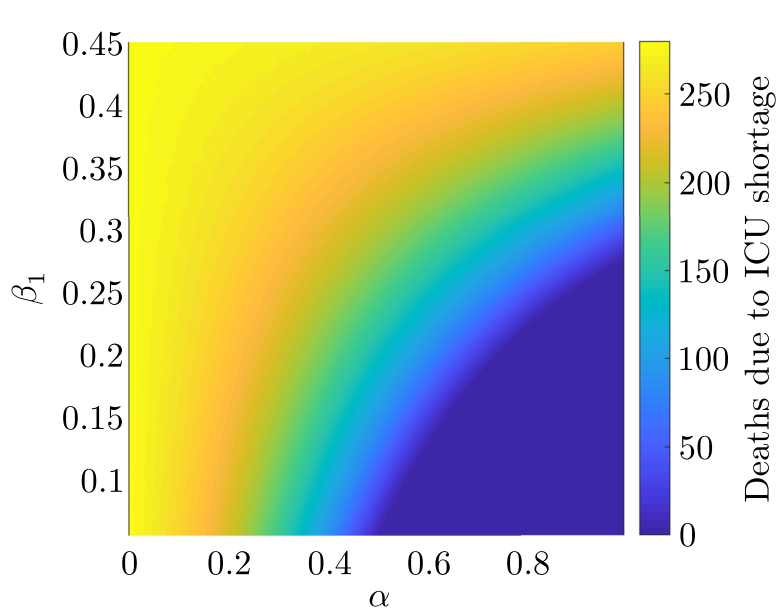

(b)

Fig. 4. (a) Temporal evolution of ICU demand and (b) number of deaths caused by ICU shortage as a function of the level, $\alpha$, and the quality, $\beta_{1}$, of the social isolation. Excepting $\beta_{1}$, the other parameters followed Table 1.

As stated, in the baseline scenario we set $\beta_{1}=\beta_{2} / 5=0.1 \mathrm{day}^{-1}$, meaning a fivefold drop in the daily contacts per person. Depending on the commitment of the individuals under social isolation, however, this rate of contact might vary significantly, altering the parameter $\beta_{1}$, i. e., the quality of the social isolation. Hence, the social isolation level, $\alpha$, must be adjusted to avoid the saturation of ICU and the mortality. We thus simulated scenarios with different values 
of $\beta_{1}$ and $\alpha$ (still set constant during the entire epidemic) to assess how the relation between these parameters affect the mortality due to ICU shortage, and the results are shown in Fig. 4. Specifically in Fig. 4a one can see the temporal evolution of the ICU demand and Fig. 4b displays the total number of deaths due to ICU shortage, both considering different values of $\alpha$ and $\beta_{1}$. It is not a surprise to see that low commitment in practicing social distancing (high values of $\beta_{1}$ ) demands higher levels of social isolation to avoid the saturation of the health system. However, one must highlight that above some values of $\beta_{1}$ there is no level of social isolation capable of avoiding deaths due to ICU shortage.

\section{Numerical experiments: Second part}

In order to investigate more realistic situations, we now take into account the inevitable lag for the population to become aware of the ongoing epidemic and then to adopt social distancing policies. We also assume the impracticability of maintaining social isolation for excessively long periods and explore its potential economic impact.

In a first approach, we looked mainly into the effect of the delay $\delta$ (see Eq. (2.8)) without specifying an end for the social isolation period, i. e., once $\alpha>0$ is adopted at $t=\delta$, it is kept constant until the end of the epidemic. Assuming a minimum of two weeks for the epidemic to be detected, we simulated several scenarios with different combinations for the parameters $\delta$ and $\alpha$, with the remaining parameters following Table 1 . The results are presented in Fig. 5 and show a region that lead to low (or none) deaths due to ICU shortage. However, one must observe that this optimal region vanishes for high values of $\delta$, meaning that hesitating to adopt social distancing policies might overburden the health system and increase the mortality rate.

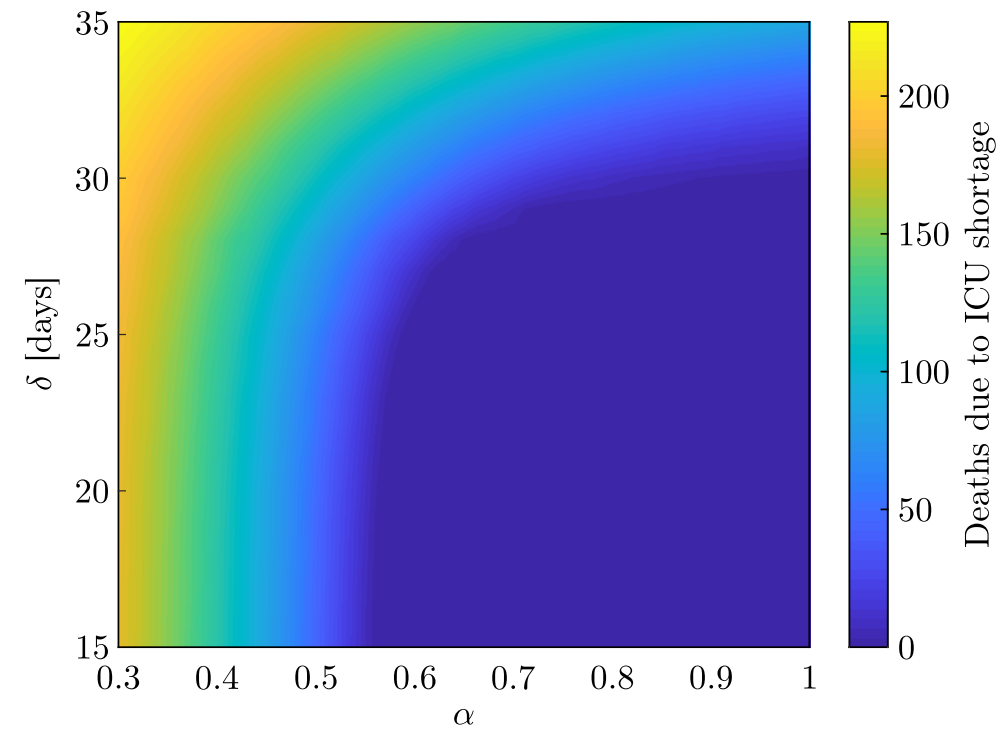

Fig. 5. Deaths caused by ICU shortage as a function of the delay, $\delta$, to implement social isolation and its level, $\alpha$ (kept constant from $t=\delta$ until the end of the epidemic). The other parameters followed Table 1.

Next, we focused on finding optimal social distancing schedules (see Eq. (2.8)) that avoid the saturation of the health system and simultaneously mitigate the economic impact. Finding a proper functional to measure such an impact, in its turn, might be quite challenging, since many factors with nonlinear dependencies between them can be taken into account. Many sectors of the economy, for example, present a tightly interdependence and, therefore, lowering the activities of 
some of them for long periods might lead to a disproportionate impact on the others. Since our goal in this experiment is not measuring the economic impact itself but only using it to probe a suitable social isolation schedule, we chose our functional as simple as possible. We assumed the economic downturn to be proportional to the number of people confined at home as well as to how long this confinement lasts. In this way, we mathematically defined the economic impact, $\xi$, as being given by the product between the level $\alpha$ of the social isolation and its duration $\tau$,

$$
\xi=\alpha \tau \text {. }
$$

Simulations were run considering specific pairs of parameters $\delta$ and $\alpha$ used in the previous experiment, namely, those whose outcomes lay roughly in the border of the region that does not present death to ICU shortage (see Fig. 5). For each pair $(\delta, \alpha)$, we searched for the minimum value of $\tau$ that guarantees zero deaths due to ICU shortage and the results are shown in Table 3. In theory, these results might help decision-makers to avoid missteps, for example, lifting social distancing too soon and inducing a peaky second wave. For example, if one hesitates 25 days to implement social distancing policy, the isolation must be carried out with level $\alpha=0.6$ and last $\tau=69$ days, in order to avoid the saturation of the health system and simultaneously mitigate the economic downturn.

Table 3. Combination of parameters $\delta, \alpha$ and $\tau$ of social distancing schedules (see Eq. (2.8)) that provide minimum economic impact $\xi$ (see Eq. (3.1)) with no death due to ICU shortage.

\begin{tabular}{cccc}
\hline$\delta$ [days] & $\alpha$ & $\tau$ [days] & $\xi$ [days] \\
\hline 15 & 0.57 & 107 & 61.0 \\
16 & 0.57 & 102 & 58.1 \\
17 & 0.57 & 98 & 56.4 \\
18 & 0.57 & 93 & 52.4 \\
19 & 0.57 & 89 & 50.7 \\
20 & 0.57 & 84 & 48.5 \\
21 & 0.57 & 79 & 45.0 \\
22 & 0.58 & 78 & 45.2 \\
23 & 0.58 & 73 & 42.3 \\
24 & 0.59 & 72 & 42.5 \\
25 & 0.60 & 69 & 42.6 \\
26 & 0.61 & 67 & 40.9 \\
27 & 0.63 & 70 & 44.1 \\
\hline
\end{tabular}

However, our simulations also show that the model is very sensitive with respect to the parameters $\delta, \alpha$ and $\tau$. Indeed, the values in Table 3 represent cliff-edge conditions, meaning that small changes in one of them might lead to very different outcomes, for instance, causing a deadly second wave. As an example, Fig. 6 shows the temporal evolution of ICU demand for two scenarios with slightly different social distancing schedules. The first one considers $\delta=15$ days, $\alpha=0.57$ and $\tau=107$ days (first row of Table 3) and presents no deaths due to ICU shortage, as shown in Fig. 6-I. In the second scenario, on the other hand, the social distancing restriction is lifted one week earlier ( $\tau=100$ days), causing a peaky second wave and the saturation of the health system, as depicted in Fig. 6-II.

Moreover, the results shown in Table 3 present a curious but understandable behavior. The longest isolation period ( $\tau=107$ days), which also corresponds to the highest economic impact

RUSSIAN JOURNAL OF NONLINEAR DYNAMICS, 2021, 17(3), 275-287 


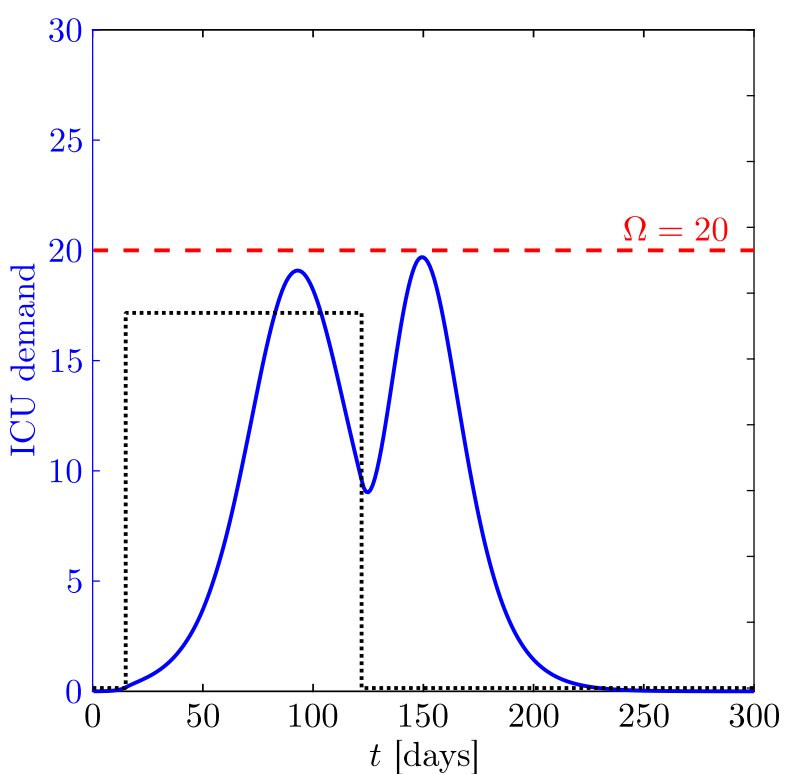

(I)

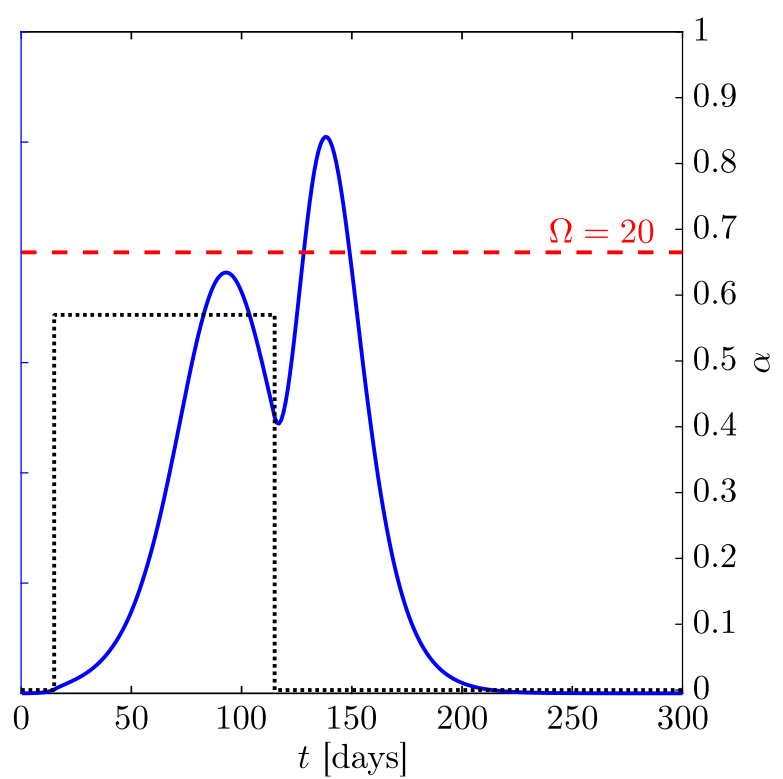

(II)

Fig. 6. Temporal evolution of ICU demand considering $\delta=15$ days, $\alpha=0.57$ and (I) $\tau=107$ days and (II) $\tau=97$ days. The remaining parameters follow Table 1 . Lifting social restrictions one week earlier in the second scenario (right) leads to a sharp second wave and the saturation of the health system.

( $\xi=61$ days $)$, matches with the shortest delay $(\delta=15$ days $)$. Put it simply, it seems that implementing social isolation too early is not the best option. It can be explained considering that, in a such case, few individuals would have contact with the virus and thus become immunized. Therefore, once the overwhelming majority of the population are kept susceptible, social restrictions must last longer, otherwise one has a sharp epidemic rebound. On the other hand, the best scenario (lowest economic impact), according to Table 3, would correspond to a social isolation with level $\alpha=0.61$, duration $\tau=67$ days and implemented with a delay $\delta=26$ days.

The extent of the economic impact, $\xi$, in its turn, has a relevant effect on the available range of the parameters that avoid the saturation of the health system. To assess this effect we simulated scenarios with three different economic impacts, $\xi=42$ days, $\xi=50$ days and $\xi=60$ days, and considering, for each one, several combinations of the parameters $\delta$ and $\tau$ ( $\alpha$ is implicit once $\xi=\alpha \tau$ ). The results are shown in Fig. 7, which presents the deaths due to ICU shortage as a function of $\delta$ and $\tau$ for each economic impact, $\xi=42$ days, $\xi=50$ days and $\xi=60$ days. One can see that the region representing few or none deaths is quite narrow in the low economic impact scenario and becomes broader for higher economic impacts. From a decision maker point of view, this finding means that focusing excessively on minimizing the economic downturn might be risky since it shortens the space for mistakes. On the contrary, accepting higher economic impacts results in broader possibilities of controlling the epidemic and avoiding the saturation of the health system.

\section{Conclusions}

Considering the SARS-Cov-2/COVID-19 pandemic context, a simple modified SIR model that incorporates social isolation and two extra compartments, hospitalized and dead due to the 


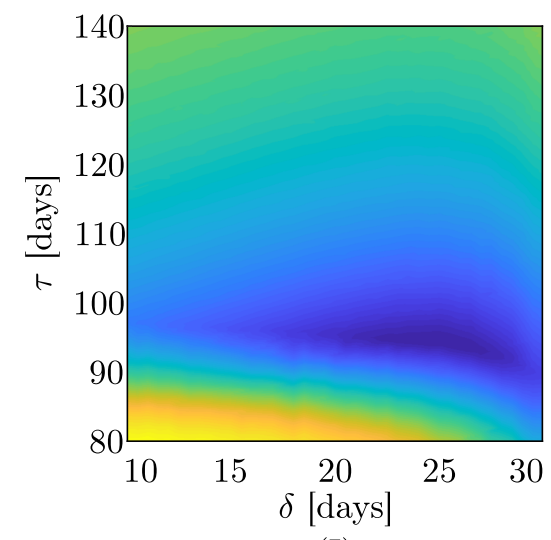

(I)

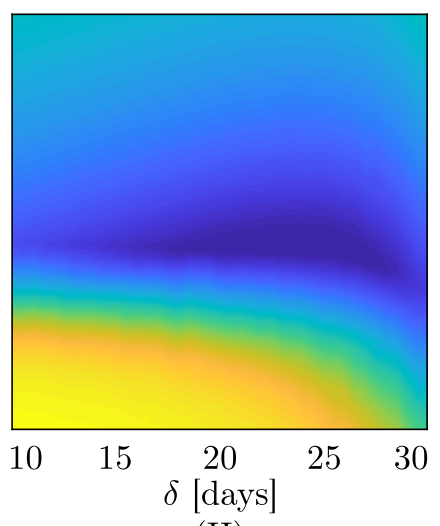

(II)

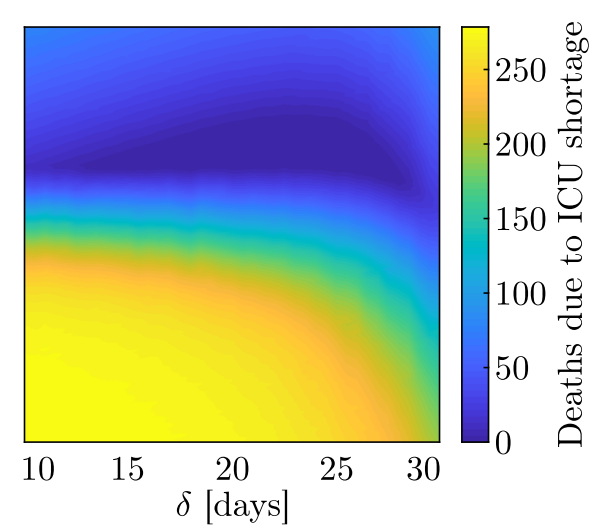

(III)

Fig. 7. Deaths due to ICU shortage as a function of $\delta$ and $\tau$ considering three scenarios with different economic impacts: (I) $\xi=42$ days, (II) $\xi=50$ days and (III) $\xi=60$ days. The region representing few or none deaths becomes broader from the lower to the higher economic impacts.

disease, was presented. In contrast to other models presented in the literature, our model also considers the rise in the mortality rate caused by ICU shortage. Numerical experiments were extensively carried out to assess how different social distancing schedules affect the mortality, especially related to the ICU shortage. Results show the striking role of suitable social distancing policies in both lowering and delaying the epidemic peak, and hence preventing the saturation of the health systems and saving lives. The potential economic impact of the social isolation policies was also investigated, and the results show that it is possible to mitigate it and, at the same time, to avoid deaths due to ICU shortage. However, the experiments also reveal that these optimal scenarios are very unstable, allowing quite narrow space for missteps. Accepting higher economic downturns, on the other hand, provides decision makers with a broader range of possibilities for implementing social isolation in order to guarantee no saturation of the health system.

We are mindful that our model has limitations. As other compartmental models, ours is also based on the assumption of a well-mixed population and, therefore, does not take into account spatial heterogeneity, which is better tackled by network models. We are also aware that demographic aspects of the populations, such as age and social stratification, could have been incorporated in the model. Nevertheless, we chose not to do so since our main goal has been designing the model to be as simple as possible so that a minimum number of equations and parameters are needed to capture the overall aspects of interest, for example, the role of the social distancing schedule in the ICU saturation and its consequent rise in the death rate. Moreover, the proposed social distancing schedule is obviously not realistic, since both its beginning and its end are abrupt and the social isolation level is kept constant during its implementation. Given this information, the results presented here should then be treated with considerable caution. Still, we hope this study might shed some light on the dark current COVID-19 battlefield and eventually the ones during future pandemics.

\section{References}

[1] Oldstone, M. B. A., Viruses, Plagues, and History: Past, Present, and Future, New York: Oxford Univ. Press, 2010. 
[2] Quammen, D., Spillover: Animal Infections and the Next Human Pandemic, New York: Norton, 2012.

[3] https://www.who.int/publications/m/item/weekly-operational-update-on-covid-19-20-november-2020 (20 Nov 2020).

[4] Kraemer, M. U. G., Yang, Ch.-H., Gutierrez, B., Wu, Ch.-H., Klein, B., Pigott, D. M., Open COVID19 Data Working Group, du Plessis, L., Faria, N.R., Li, R., Hanage, W. P., Brownstein, J. S., Layan, M., Vespignani, A., Tian, H., Dye, Ch., Pybus, O. G., and Scarpino, S. V., The Effect of Human Mobility and Control Measures on the COVID-19 Epidemic in China, Science, 2020, vol. 368, no. 6490, pp. 493-497.

[5] https://www.who.int/publications/m/item/situation-report-10 (30 Jan 2020).

[6] World Health Organization, Coronavirus Disease 2019 (COVID-19): Situation Report-51, see https://www.who.int/docs/default-source/coronaviruse/situation-reports/20200311-sitrep-51-covid19.pdf?sfvrsn=1ba62e57_10 (11 March 2020).

[7] Cui, J., Li, F., and Shi, Z. L., Origin and Evolution of Pathogenic Coronaviruses, Nat. Rev. Microbiol., 2019, vol.17, no. 3, pp. 181-192.

[8] Kissler, S. M., Tedijanto, Ch., Goldstein, E., Grad, Y.H., and Lipsitch, M., Projecting the Transmission Dynamics of SARS-CoV-2 through the Postpandemic Period, Science, 2020, vol. 368, no. 6493, pp. 860-868.

[9] Iyengar, K., Bahl, S., Vaishya, R., and Vaish, A., Challenges and Solutions in Meeting Up the Urgent Requirement of Ventilators for COVID-19 Patients, Diabetes Metab. Syndr. Clin. Res. Rev., 2020, vol. 14, no. 4, pp. 499-501.

[10] Qiu, H., Tong, Zh., Ma, P., Hu, M., Peng, Zh., Wu, W., Du, B., and China Critical Care Clinical Trials Group (CCCCTG), Intensive Care during the Coronavirus Epidemic, Intensive Care Med., 2020, vol. 46, no. 4, pp. 576-578.

[11] Anderson, R. M., Heesterbeek, H., Klinkenberg, D., and Hollingsworth, T. D., How Will CountryBased Mitigation Measures Influence the Course of the COVID-19 Epidemic?, The Lancet, 2020, vol. 395, no. 10228, pp. 931-934.

[12] Kenyon, C., Flattening-the-Curve Associated with Reduced COVID-19 Case Fatality Rates- an Ecological Analysis of 65 Countries, J. Infect., 2020, vol. 81, no. 1, e98-e99.

[13] Bauch, C. T., Estimating the COVID-19 R Number: A Bargain with the Devil?, Lancet Infect. Dis., 2021, vol. 21, no. 2, pp. 151-153.

[14] Li, Y., Campbell, H., Kulkarni, D., Harpur, A., Nundy, M., Wang, X., Nair, H., and Usher Network for COVID-19 Evidence Reviews (UNCOVER) Group, The Temporal Association of Introducing and Lifting Non-Pharmaceutical Interventions with the Time-Varying Reproduction Number (R) of SARS-CoV-2: A Modelling Study across 131 Countries, Lancet Infect. Dis., 2021, vol. 21, no. 2, pp. 193-202.

[15] Thunström, L., Newbold, S.C., Finnoff, D., Ashworth, M., and Shogren, J.F., The Benefits and Costs of Using Social Distancing to Flatten the Curve for COVID-19, J. Benefit-Cost Analysis, 2020, vol. 11, no. 2, pp. 179-195.

[16] Nicola, M., Alsaf, Z., Sohrabi, C., Kerwan, A., Al-Jabir, A., Iosifidis, Ch., Agha, M., and Agha, R., The Socio-Economic Implications of the Coronavirus Pandemic (COVID-19): A Review, Int. J. Surg. Open, 2020, vol. 78, pp. 185-193.

[17] COVID-19 in Brazil: "So What?", The Lancet, 2020, vol. 395, no. 10235, p. 1461.

[18] Vespignani, A., Tian, H., Dye, C., Smith, J. O. L., Eggo, R. M., Shrestha, M., Scarpino, S. V., Gutierrez, B., Kraemer, M. U. G., Wu, J., Leung, K., and Leung, G. M., Nature Rev. Phys., 2020, vol. 2, no. 6, pp. 279-281.

[19] Nakamura, G., Grammaticos, B., and Badoual, M., Confinement Strategies in a Simple SIR Model, Regul. Chaotic Dyn., 2020, vol. 25, no.6, pp. 509-521. 
[20] Pedro, S.A., Ndjomatchoua, F. T., Jentsch, P., Tchuenche, J. M., Anand, M., and Bauch, Ch. T., Conditions for a Second Wave of COVID-19 due to Interactions between Disease Dynamics and Social Processes, Front. Phys., 2020, vol. 8, Art. 5/4514, 9 p.

[21] Block, P., Hoffman, M., Raabe, I. J., Dowd, J. B., Rahal, C., Kashyap, R., and Mills, M. C., Social Network-Based Distancing Strategies to Flatten the COVID-19 Curve in a Post-Lockdown World, Nat. Hum. Behav., 2020, vol. 4, pp. 588-596.

[22] Flaxman, S., Mishra, S., Gandy, A., Unwin, H. J. T., Coupland, H., Mellan, T. A., Zhu, H., Berah, T., Eaton, J. W., Guzman, P. N. P., et al., Report 13: Estimating the Number of Infections and the Impact of Non-Pharmaceutical Interventions on COVID-19 in 11 European Countries, Imperial College London, https://spiral.imperial.ac.uk/handle/10044/1/77731 (2020).

[23] Kucharski, A. J., Russell, T. W., Diamond, Ch., Liu, Y., Edmunds, J., Funk, S., Eggo, R. M., on behalf of the Centre for Mathematical Modelling of Infectious Diseases COVID-19 Working Group, Lancet Infect. Dis., 2020, vol. 20, no. 5, pp. 553-558.

[24] Kermack, W. O. and McKendrick, A. G., Contributions to the Mathematical Theory of Epidemics, Proc. Roy. Soc. Edinburgh Sect. A, 1927, vol. 115, no. 772, pp. 700-721.

[25] Keeling, M. J. and Rohani, P., Modeling Infectious Diseases in Humans and Animals, Princeton: Princeton Univ. Press, 2007.

[26] Fine, P., Eames, K., and Heymann, D. L., "Herd Immunity": A Rough Guide, Clin. Infect. Dis., 2011, vol. 52, no. 7, pp. 911-916.

[27] Mossong, J., Hens, N., Jit, M., Beutels, P., Auranen, K., Mikolajczyk, R., Massari, M., Salmaso, S., Tomba, G.S., Wallinga, J., Heijne, J., Sadkowska-Todys, M., Rosinska, M., and Edmunds, W. J., Social Contacts and Mixing Patterns Relevant to the Spread of Infectious Diseases, PLoS Med., 2008, vol. 5, no. 3, e74, 11 p.

[28] Prin, M. and Wunsch, H., International Comparisons of Intensive Care: Informing Outcomes and Improving Standards, Curr. Opin. Crit. Care, 2012, vol. 18, no.6, pp. 700-706.

[29] Rhodes, A., Ferdinande, P., Flaatten, H., Guidet, B., Metnitz, P. G., and Moreno, R. P., The Variability of Critical Care Bed Numbers in Europe, Intensive Care Med., 2012, vol. 38, no. 10, pp. 16471653. 